\title{
Die Diagnose eines Schwangerschaftsdiabetes hat ihre Tücken
}

\author{
Ein Gestationsdiabetes (GDM) gehört zu den häufigsten Komplikationen in der Schwangerschaft. \\ Der Nachweis ist über die Nüchternglukosewerte bzw. einen oralen Glukosetoleranztest (oGGT) \\ im Prinzip einfach, aber eine einzelne Messung ist fehleranfällig.
}

Man sollte sich bei der Diagnose eines Schwangerschaftsdiabetes nicht auf Einzelwerte verlassen, sagte Dr. Guido Freckmann vom Institut für Diabetes-Technologie an der Universität Ulm beim Diabetes-Kongress in Hamburg.

In der DDG-Praxisleitlinie unterscheidet sich die diagnostische Vorgehensweise vor und nach der 24. Schwangerschaftswoche. Vor der 24. SSW wird nach einer auffälligen Gelegenheits-Glukose $\geq 200 \mathrm{mg} / \mathrm{dl}$ (oder Nüchternglukose $\geq 92 \mathrm{mg} / \mathrm{dl}$ ) eine Zweitmessung empfohlen. Ist der Nüchternwert $\geq 92 \mathrm{mg} /$ dl liegt ein GDM, bei Werten darüber ein manifester Diabetes vor. Nach der 24. SSW wird ein 75-Gramm-oGGT empfohlen, nüchtern unter standardisierten Bedingungen und qualitätsgesicherter Glukosemessung aus venösem Blut, eventuell nach einem Screening mittels 50-Gramm-Suchtest (Grenzwert $\geq 135$ $\mathrm{mg} / \mathrm{dl}$ nach 1 Stunde). Bei Überschreiten von mindestens einem Grenzwert (nüchtern $\geq 92 \mathrm{mg} / \mathrm{dl}$, nach 1 Stunde $\geq 180 \mathrm{mg} / \mathrm{dl}$, nach 2 Stunden $\geq 153 \mathrm{mg} / \mathrm{dl}$ ) kann die Diagnose GDM gestellt werden, hier also nach einmaliger Messung.

\section{Die Crux liegt bei der Blutentnahme}

Freckmann, selbst Diabetologe, hob die Fehlerquellen bei der Messung hervor. „Die Crux liegt bei der Blutentnahme“, sagte er. Wegen des Glukoseabbaus durch anaerobe Glukolyse (-7 \% pro Stunde) müsse die Messung nach venöser Blutentnahme innerhalb weniger Minuten erfolgen oder eine Glykolysehemmung sichergestellt werden, etwa durch Fluoridzitrat EDTA Röhrchen, die strikt nach den Herstelleranweisungen benutzt werden sollten. Besonders bei grenzwertnahen Glukosekonzentrationen können Fehler bei der präanalytischen Probenhandhabung die Diagnose eines GDM beeinflussen und sich auf Gesundheit von Mutter und Kind auswirken, warnte Freckmann. Er sieht weiteres Verbesserungspotenzial: Die maximalen Abweichungen vom Zielwert von $11 \%$, die in der Qualitätssicherung der Glukosemessung nach Rili-BÄK (Richtlinie der Bundesärztekammer zur Qualitätssicherung Laboratoriums-medizinischer Untersuchungen) genannt sind, seien sehr hoch. „Für die Diagnostik sind engere Diagnosegrenzen wünschenswert“, so Freckmann. Er riet, bei Werten im Bereich der messtechnischen Unsicherheit erneut zu messen und andere Befunden für die Diagnose hinzuzuziehen. Auch sollte überprüft werden, ob POCT-Unit-Use-Systeme für die GDM-Diagnose geeignet seien.

Welche weiteren Parameter zur Beurteilung des Glukosestoffwechsels sollten bei Schwangeren erhoben werden? Zwar ist die $\mathrm{HbA}_{1 \mathrm{c}}$-Bestimmung laut DDG-Leitlinie wegen der geringen Sensitivität nicht zum GDM-Screening und auch nicht zur Therapiekontrolle geeignet, berichtete Dr. Christoph Neumann, niedergelassener Diabetologe in München. Aber der Wert sollte zur Diagnose eines manifesten Diabetes herangezogen und auch zum Zeitpunkt der GDM-Diagnose gemessen werden. Bei Dia- betikerinnen sollte der $\mathrm{HbA}_{1 \mathrm{c}}$ in der Schwangerschaft alle 4-6 Wochen bestimmt werden.

Diabetes-Antikörper können zur Risikostratifizierung von Schwangeren mit GDM herangezogen werden. Bei Nachweis von Antikörpern gegen GAD und/oder IA2 liegt das postpartum Diabetesrisiko der Frauen bei über $90 \%$ im Vergleich zur nur $14 \%$ bei Frauen ohne Antikörper, berichtete Neumann. Weitere Risikofaktoren für das Auftreten eines manifesten Diabetes sind Insulintherapie, Body Mass Index $>30$ $\mathrm{kg} / \mathrm{m}^{2}$ und mehr als zwei vorangegangene Schwangerschaften. Auch die Vorhersage eines Typ-1-Diabetes wird durch Bestimmung des Antikörperstatus verbessert. 3 positive Antikörper (GAD, IA2, ICA) wiesen mit einer Sensitivität von $82 \%$ auf einen bevorstehenden Typ-1-Diabetes hin, so Neumann. Fruktosami-

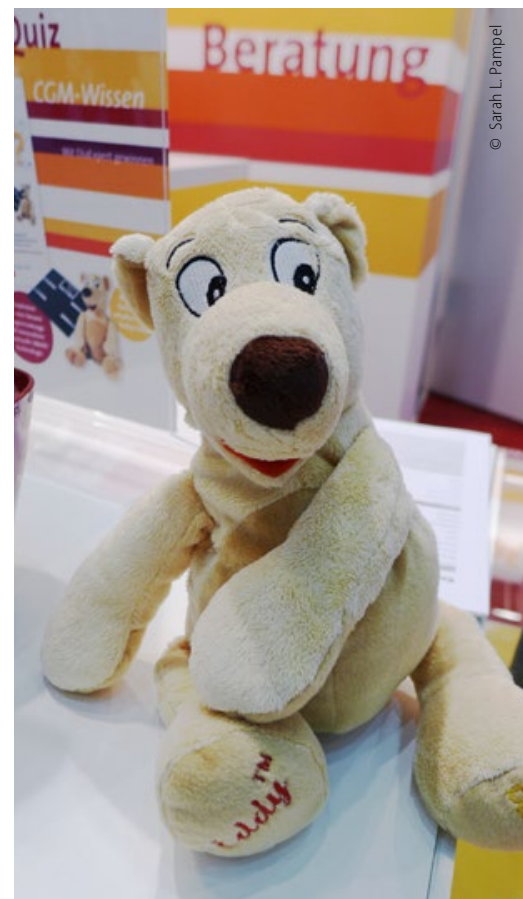

Auch bei Gestationsdiabetes ist Beratung besonders wichtig. ne, deren Konzentration proportional zum Glukosegehalt im Blut steigt, sind zur GDM-Diagnose ebenfalls nicht geeignet, aber vermutlich ein besserer Indikator der Glykämie bei GDM und Diabetes in der Schwangerschaft als der $\mathrm{HbA}_{1 \mathrm{c}}$. Fructosamine (Normbereich 200-290 $\mu$ mol/l) geben Auskunft über die mittleren Blutzuckerwerte in den letzten 1-3 Wochen.

Der Anstieg von Ketonen ist in der Schwangerschaft physiologisch. Bei rund $30 \%$ aller Schwangeren (mit und ohne Diabetes) können im ersten Morgenurin Ketone nachgewiesen werden, berichtete Neumann. Ein Monitoring werde empfohlen, obwohl unklar sei, ob sich Ketone auf den Verlauf der Schwangerschaft ungünstig auswirken. Ein erhöhter hoch sensitiver CRP-Wert und ein erniedrigtes C-Peptid sind nach Angaben von Neumann sensitive Marker zur Vorhersage eines GDM. Der positive prädiktive Wert liege bei rund $60 \%$. Ein neuer Biomarker für eine schwangerschaftsinduzierte Glukosetoleranz ist glykiertes CD59 im Plasma. Der Wert sei bei Frauen mit gesichertem GDM 10fach erhöht und könnte künftig eventuell den oGGT ablösen, so Neumann. Last not least: Bei Frauen mit Glukosetoleranzstörung in der Schwangerschaft und positiver Familienanamnese für einen „milden“ Typ-2-Diabetes sollte auch an einen monogenen MODY 2-Diabetes gedacht werden.

Roland Fath

Quelle: Symposium „Schwangerschaft + Technologie“ beim Diabeteskongress am 26. Mai 2017 in Hamburg 